\title{
ESTUDIO
}

\section{TIERRA, COMUNIDAD E IDENTIDAD MAPUCHE}

\author{
Eduardo Valenzuela
}

\begin{abstract}
En este artículo se examinan brevemente los datos de identificación étnica que contiene la encuesta realizada por el Centro de Estudios Públicos en población mapuche. Los resultados del estudio indican que los factores más determinantes de esta identidad son la lengua y la tierra. La información muestra un deterioro notable de la lengua mapuche que va acompañada también de una desarticulación profunda de la comunidad ritual y de la comunidad de sangre. La posibilidad de afirmar una identidad étnica en estos factores aparece muy disminuida, pero se recupera notablemente en la referencia a la tierra que se ofrece como último soporte de una identidad étnica duramente desafiada de todos modos por los actuales procesos de asimilación urbana.
\end{abstract}

Eduardo Valenzuela. D.E.A., École des Hautes Etudes en Sciences Sociales (Francia). Director del Instituto de Sociología, Pontificia Universidad Católica de Chile. Autor de numerosos escritos en teoría sociológica, sociología de la cultura y sociología comparada.

N. del E.: Sobre los resultados de la encuesta mapuche realizada por el CEP (2006), véanse también en esta edición los trabajos de Fernando Zúñiga, Ignacio Irarrázaval y M. de los Ángeles Morandé, Aldo Mascareño, Rolf Foerster y Sonia Montecino, Juan M. Ossio, así como el comentario de Jorge Larraín al artículo de Aldo Mascareño y la presentación de Carolina Segovia y Lucas Sierra. 


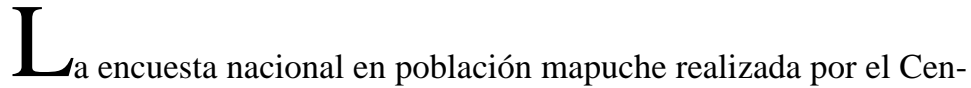
tro de Estudios Públicos $(2006)^{1}$ tiene un novedoso diseño muestral: ha tomado como base muestral las comunas con mayor densidad de población mapuche según el último censo y ha construido muestras en área urbana y rural de mapuches (según el mismo formato de autoidentificación que ocupó el censo) y no mapuches, que comprende a vecinos o corresidentes que no se identifican como mapuches. Después de usar el criterio censal de autoidentificación étnica para seleccionar las diferentes muestras, el cuestionario vuelve a interrogar acerca de la identidad con una pregunta de tinte más subjetivo, “¿se siente Ud. mapuche, chileno o una mezcla de los dos?”. Esta última pregunta que precisa la intensidad de la identificación étnica en población mapuche es la que se toma como base de este análisis que pretende entregar información y alguna reflexión acerca de la naturaleza y alcance de la identidad mapuche en la sociedad contemporánea.

\section{Identidad}

Las declaraciones de autoidentificación según el formato del cuestionario muestran un cierto balance entre la afirmación étnica ("mapuche" a secas, 38\%) y la afirmación mixta ("chileno y mapuche al mismo tiempo", 37\%). Cuando se incluye la precedencia, "mapuche primero, chileno después”, la afirmación étnica alcanza al 53\% y la afirmación mixta (“chileno primero, mapuche después”) al 43\% (con una pequeñísima proporción de $3 \%$ de mapuches que se identifica exclusivamente como chileno). Esta autoidentificación étnica está fuertemente determinada por la lengua y la ruralidad según muestran los resultados del análisis de regresión logística que se presentan más abajo. La probabilidad de identificarse como mapuche a secas aumenta 4,8 veces entre quienes habitan en áreas rurales respecto de quienes lo hacen en áreas urbanas y entre quienes hablan la lengua mapuche respecto de quienes no lo hacen. Ambos factores - hablar la lengua y vivir en la tierra - están muy por encima de cualquier otro: la edad (tener 35 años o más) no aparece significativamente asociada con la identidad, mientras que la baja escolaridad (hasta 8 años de estudio) tiene una asociación modestamente positiva. La identificación religiosa, sea católica o evangélica disminuye la probabilidad de identificarse como mapuche respecto de quienes no declaran ninguna religión aunque también de un modo que no re-

\footnotetext{
${ }^{1}$ Centro de Estudios Públicos (CEP): "Estudio de Opinión Pública: Los Mapuche Rurales y Urbanos Hoy. Mayo 2006”. Santiago: CEP, 2006.
} 
CUADRO N ${ }^{\circ}$ 1: MODELO DE REGRESIÓN LOGÍSTICA PARA PROBABILIDAD DE IDENTIFICARSE COMO MAPUCHE

\begin{tabular}{lrrrr}
\hline & B & E.T. & Sig. & Exp(B) \\
\hline & $-0,812$ & 0,181 & 0,000 & 0,444 \\
Católico & $-0,472$ & 0,188 & 0,012 & 0,624 \\
Evangélico & 1,583 & 0,130 & 0,000 & 4,870 \\
Habita zona rural & 0,172 & 0,134 & 0,201 & 1,188 \\
35 o más años de edad & 0,512 & 0,138 & 0,000 & 1,669 \\
Hasta 8 años de escolaridad & & & & \\
$\begin{array}{l}\text { Habla lengua mapuche igual o } \\
\text { mejor que el castellano }\end{array}$ & 1,561 & 0,195 & 0,000 & 4,765 \\
Constante & $-1,231$ & 0,169 & 0,000 & 0,292 \\
& & & & \\
N: 1487 & & & & \\
\hline
\end{tabular}

$\mathrm{R}^{2}$ Nagelkerke: 0,323

Hosmer y Lemeshow: 0,784

sulta decisivo. La identidad étnica tiene como fundamento dos elementos extremadamente inestables en la sociedad mapuche contemporánea: la lengua y la tierra, ambos muy desafiados por los procesos de asimilación urbanos.

\section{Lengua}

El examen del fundamento cultural de la identidad étnica remite en primer lugar al estado de la lengua mapuche. Según los datos que ofrece la encuesta, el $15 \%$ de los mapuches habla su lengua igual o mejor que el castellano (hasta el 28\% de los mapuches del área rural, solamente 6\% de los mapuches en área urbana). También existen diferencias de edad muy pronunciadas: $35 \%$ de los adultos en áreas rurales habla la lengua mapuche, cosa que ocurre solamente con el $17 \%$ de los jóvenes mapuches rurales. Entre los jóvenes mapuches urbanos la lengua se ha perdido casi completamente. Es importante observar que la lengua sobrevive algo mejor en hombres que en mujeres (32\% en hombres del área rural y $24 \%$ en mujeres) y en mapuches de baja escolaridad (hasta 32\% en mapuches con 3 años o menos de escolaridad, contra apenas un $2 \%$ en mapuches con escolaridad universitaria, diferencia que no se explica solamente por la asociación entre ruralidad y baja escolaridad). La lengua viva es todavía algo menor. Alrededor del $80 \%$ de los mapuches rurales que conocen la lengua igual o mejor que el castellano la habla asiduamente (al menos una vez a la semana), lo que 
entrega una proporción de 22\% de mapuches en áreas rurales que conocen y hablan la lengua. En áreas urbanas solamente la mitad tiene oportunidad de hablar con frecuencia la lengua mapuche, lo que permite estimar en apenas 3\% la proporción de mapuches que conoce y habla la lengua en la ciudad. El declive de la lengua por desuso es muy importante en las ciudades: se puede estimar en alrededor del $20 \%$ los mapuches que conocen pero no usan la lengua en la ciudad (nunca o casi nunca, o solo en ocasiones muy especiales) ${ }^{2}$.

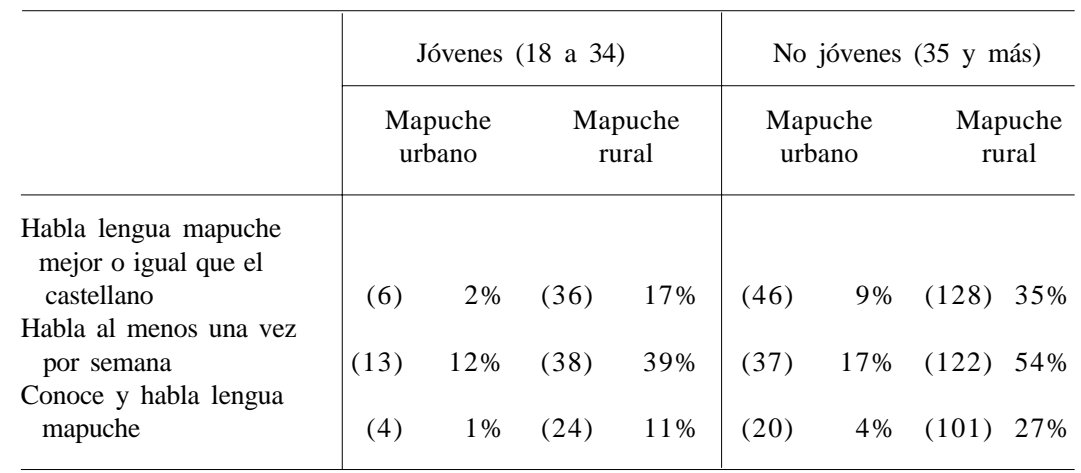

\section{Religión}

Todavía más importante que el declive de la lengua es el estado de lo que usualmente se llama cultura ancestral. En la encuesta se registran niveles altos de conocimiento de lo que significa machitún (ritual de curación o sanación) y ngillatun (ceremonia para obtener una buena cosecha u otro beneficio) y algo menos del wetripantu (fiesta del año nuevo mapuche, que corresponde al día de San Juan). No obstante, el conocimiento de los llamados ritos de pasaje es extremadamente bajo: solamente el 10\% de los mapuches sabe qué es lakutun (ceremonia en la cual se pone nombre a los niños) y ngapin (ceremonia por la cual dos personas se unen matrimonialmente) y el $15 \%$ acierta en definir eluwun (rito para despedir a los muertos). Las

${ }^{2}$ Un análisis más preciso sobre el estado de la lengua mapuche se ofrece en el artículo de Fernando Zúñiga en este mismo número de Estudios Públicos. 
CUADRO No 3: CONOCIMIENTO DE CULTURA ANCESTRAL SEGÚN RELIGIÓN Y HÁBITO RELIGIOSO: MAPUCHES URBANOS Y RURALES

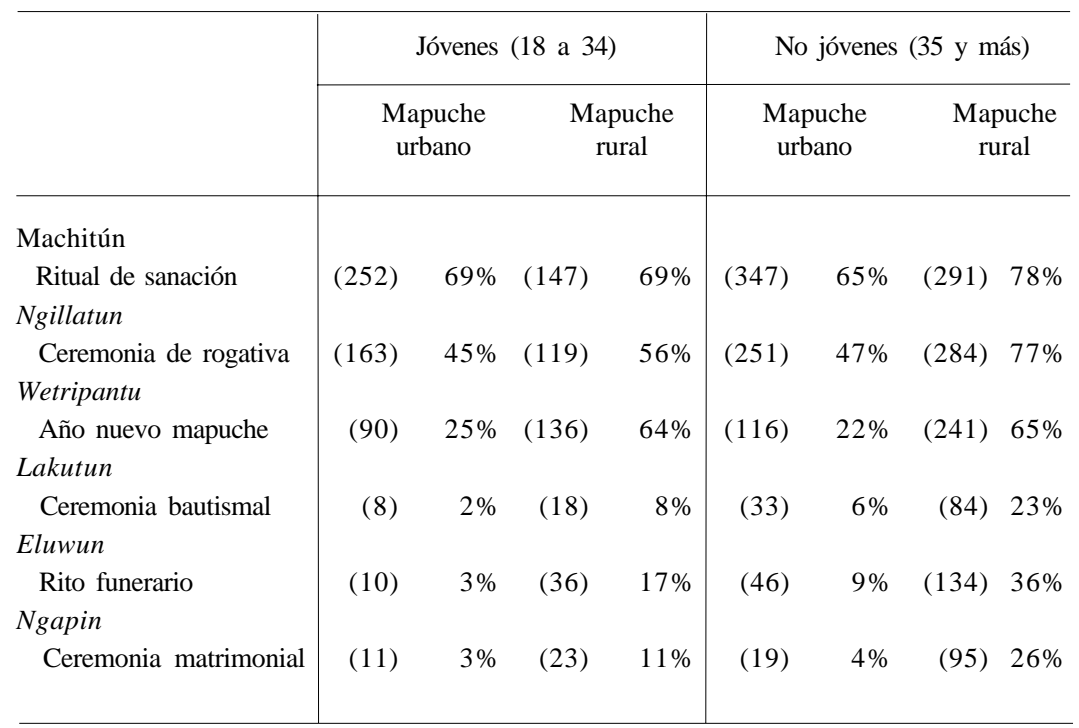

proporciones máximas se alcanzan en áreas rurales con 17\%, 20\% y 29\% respectivamente, mientras que en áreas urbanas estos ritos de pasaje son casi completamente desconocidos. La participación en estos ritos es prácticamente inexistente: la encuesta sólo entrega información acerca de la proporción que declara haberse casado según la costumbre mapuche (hasta $23 \%$ en área rural) mientras que los ritos bautismales y funerarios se consideran extinguidos. Los ritos de pasaje son la arquitectura religiosa de cualquier comunidad que se constituye y actualiza constantemente en la celebración de sus nuevos miembros, en consagración de las alianzas y en la despedida de sus muertos. Los ritos de pasaje son asimismo el verdadero sostén de la lengua que adquiere en ellos el carácter de lengua sagrada o ceremonial, el momento en que una comunidad es capaz de observarse y pronunciarse a sí misma en su lengua original. La evidencia muestra que estos ritos han sido largamente cristianizados y se pronuncian en lengua castellana, tal como lo hacen los demás chilenos. Es probable que el sentimiento de integración nacional (73\% de los mapuches dice sentirse plenamente integrado al país) descanse en esta realidad fundamental: los mapuches nacen, se casan y mueren como los demás chilenos, en los mar- 
cos de ritos y ceremonias semejantes y de una lengua común ${ }^{3}$. Estos datos sugieren que la identidad mapuche no se constituye por referencia a una comunidad: lo que sobrevive de cultura ancestral es el machitún (ritual de sanación, que como todo vínculo mágico es más bien privado) y el ngillatun, la única ceremonia comunitaria vinculada a la fertilidad de la tierra, con dificultades para actualizarse fuera del campo y de la economía rural. El ngillatun es una ceremonia vigente en el campo, $45 \%$ de los mapuches rurales declara participar con frecuencia o a veces en un ngillatun, pero la participación del mapuche urbano desciende bruscamente a 14\%. La machi asimismo es muy poco solicitada incluso en el campo (25\%) para desaparecer casi completamente en la ciudad (5\%).

\section{Matrimonio}

Esta ausencia de comunidad se manifiesta también en la importancia del connubio o del matrimonio mixto en la población mapuche. Entre mapuches urbanos, el $57 \%$ de los matrimonios o convivencias son definitivamente mixto (mapuches con chilenos), aunque entre mapuches rurales el connubio baja a $22 \%$. La diferencia urbano-rural en prevalencia de matrimonios mixtos es muy pronunciada: las tasas de homogamia en área urbana (mapuche con mapuche) alcanzan a $23 \%$ y en área rural suben a $65 \%$. Es importante notar, sin embargo, que 38\% de no mapuches rurales declara un matrimonio con mapuches (contra apenas un $4 \%$ de no mapuches urbanos) lo que hace más abierta la sociedad rural de lo que aparece a primera vista. Los datos muestran también que no hay ninguna diferencia importante entre hombres y mujeres: unos y otras se casan más o menos en la misma proporción con pares o no pares. También sucede que el connubio aumenta en las generaciones más jóvenes tanto en área rural como urbana aunque con una brecha moderada de 10 puntos en los extremos de edad, y se incrementa sobre todo con la escolaridad con una brecha enorme: la homo-

${ }^{3}$ La identificación religiosa de los mapuches no tiene un sesgo étnico como habitualmente se cree: los mapuches tienden a compartir la identidad religiosa de la población no mapuche de su misma condición. La proporción de mapuches que se declara católico (49\%) es significativamente menor que el promedio nacional (70\% según último censo), aunque la proporción de no mapuches corresidentes que se declara católico es bastante similar (56\%). Inversamente, la proporción de evangélicos dobla el promedio nacional alcanzando al 35\% entre mapuches y solamente algo menos, 27\%, entre no mapuches. La estructura de la identificación religiosa entre mapuches y no mapuches en áreas urbanas es exactamente la misma, mientras que en áreas rurales los mapuches adhieren algo más que los no mapuches al pentecostalismo y algo menos al catolicismo. 
gamia entre mapuches con alta escolaridad es de 13\% mientras que en mapuches con baja escolaridad alcanza a $61 \%$, diferencia que no se explica solamente por la asociación entre baja escolaridad y ruralidad.

Los resultados acerca de matrimonio mixto concuerdan ampliamente con la actitud respectiva: $65 \%$ de los mapuches considera que está bien que su hija o hermana se case con una persona no mapuche y solamente un $6 \%$ lo considera definitivamente mal. Esta disposición es apenas más baja en área rural (61\% con 12\% de desaprobación, hasta un máximo de 15\% entre los más ancianos) donde sin embargo la homogamia es mucho más frecuente. También es importante mencionar que la aprobación del connubio es muy similar en mapuches y no mapuches corresidentes: la disposición de la población mapuche a casarse con no mapuches es la misma que la disposición de sus vecinos no mapuches a casarse con mapuches. La aceptación del matrimonio mixto reaparece con la frase "está bien que una persona mapuche se case con una persona no mapuche” que obtiene una proporción de $75 \%$ de acuerdo que puede llegar a 79\% en área urbana y bajar hasta 69\% en área rural ligeramente más reticente. Esta amplísima aceptación del connubio confirma que la identidad mapuche no está afincada en la comunidad, en el sentido convencional de una comunidad de sangre, que aparece por el contrario enteramente abierta y dispuesta al intercambio exogámico. Muchas comunidades, sobre todo aquellas que han perdido la tierra como el pueblo judío, se afirman justamente en la prohibición del connubio y en reglas de comensalidad que dificultan el contacto con extraños, cosas que no existen en absoluto en este caso. Estos mecanismos de autosegregación, completados muchas veces con otros de segregación o

CUADRO N ${ }^{\circ}$ 4: HOMOGAMIA EN POBLACIÓN MAPUCHE Y NO MAPUCHE: PROPORCIÓN DE HOMBRES Y MUJERES QUE IDENTIFICAN A SU CÓNYUGE COMO MAPUCHE, MAPUCHE Y CHILENO, Y CHILENO, SEGÚN RESIDENCIA URBANO-RURAL

\begin{tabular}{|c|c|c|c|c|c|c|}
\hline & $\begin{array}{l}\text { Mapuche } \\
\text { rural }\end{array}$ & $\begin{array}{c}\text { Mapuche } \\
\text { urbano }\end{array}$ & $\begin{array}{c}\text { Total } \\
\text { mapuche }\end{array}$ & $\begin{array}{c}\text { No } \\
\text { mapuche }\end{array}$ & $\begin{array}{c}\text { No } \\
\text { mapuche }\end{array}$ & $\begin{array}{l}\text { Total no } \\
\text { mapuche }\end{array}$ \\
\hline \multicolumn{7}{|l|}{ HOMBRES } \\
\hline Mapuche & 68 & 21 & 39 & 36 & 3 & 16 \\
\hline Mapuche y chileno & o 12 & 19 & 16 & 11 & 8 & 10 \\
\hline Chileno & 20 & 61 & 45 & 53 & 89 & 74 \\
\hline \multicolumn{7}{|l|}{ MUJERES } \\
\hline Mapuche & 62 & 25 & 38 & 40 & 5 & 19 \\
\hline Mapuche y chileno & o 14 & 21 & 19 & 10 & 6 & 8 \\
\hline Chileno & 24 & 54 & 44 & 50 & 88 & 74 \\
\hline
\end{tabular}


discriminación abierta, han fundado comunidades solidísimas y que se mantienen intactas en medio de las mayores perturbaciones. El pueblo mapuche ofrece una imagen distinta: la posibilidad de afirmar la identidad en una comunidad de lengua o de sangre parecen desvanecerse completamente, aunque todavía subsiste la tierra como último soporte de una identidad viva y actual.

\section{Tierra}

Todos los datos de la encuesta coinciden en la afirmación de la tierra como el asiento fundamental de la identidad mapuche. Después de la lengua (51\%), "vivir del trabajo de la tierra" es el segundo elemento que los mapuches consideran más importante para mantener su cultura (32\%), muy por encima de "casarse entre mapuches" (14\%) y "participar en ceremonias o ritos" (15\%), lo que vuelve a mostrar que la cultura ha perdido su soporte comunitario. En el plano de las políticas públicas este orden entre la lengua y la tierra se invierte: la "recuperación de tierras indígenas ancestrales" (35\%) es el principal problema al que el gobierno debería dedicar su atención, muy por delante de la "protección y fomento de la lengua mapuche" (17\%). Cuando se pregunta por la forma en que el país debe reparar o compensar a los mapuches, las tierras vuelven a ocupar el lugar primero (48\%), mientras que el "fomento de la lengua y cultura” ocupa una posición mucho más discreta (6\%). La reparación por tierras prima por sobre la educación (19\%) y el empleo (10\%) y se sitúa largamente por encima del reconocimiento social y legal de la etnia mapuche (14\%). La tierra es la modalidad de reparación preferida sobre todo por los mapuches urbanos (59\%) que se ubican por arriba de los mapuches rurales en este aspecto (39\%). Totalmente decisiva es la opinión acerca de la capacidad de mantener la cultura mapuche en el medio urbano: $76 \%$ de los mapuches considera que quienes viven en la ciudad pierden su contacto con la cultura mapuche. La urbanización es vista como una amenaza cultural de primer orden justamente por su impacto sobre la lengua y sobre todo por la pérdida de contacto con la tierra. La incomodidad urbana de la población mapuche es manifiesta: por ejemplo, 66\% considera que se vive mejor en el campo contra solamente $22 \%$ que afirma que se está mejor en la ciudad. Esta apreciación es más fuerte entre mapuches rurales, $79 \%$ declara que se vive mejor en el campo, pero se mantiene alta todavía entre mapuches urbanos (58\%). Asimismo, la disposición a irse a vivir al campo entre mapuches urbanos alcanza a $71 \%$ (con un $46 \%$ que marca la alternativa más vehemente, le gustaría mucho 
radicarse en el campo), mientras un 69\% de los mapuches rurales rechaza la posibilidad de vivir en la ciudad. El horizonte rural del mapuche urbano se mantiene intacto entre los jóvenes (68\% le gustaría vivir en el campo), entre los cuales debe contarse una proporción importante de migrantes de segunda generación que no obstante prefieren la vida rural.

En este contexto de afirmación identitaria de la tierra no debe sorprender demasiado la justificación del "uso de la fuerza para reclamar tierras" que alcanza a $22 \%$ entre quienes la justifican incondicionalmente y $36 \%$ en algunas circunstancias (aunque un 39\% no justifica el uso de la fuerza, lo que muestra que se trata de un tema controversial dentro de la población mapuche). Esta legitimación de la violencia étnica no tiene los sesgos de edad y escolaridad que son característicos del radicalismo político, usualmente alojado en jóvenes de alta escolaridad relativa. Al contrario, la justificación del uso de la fuerza está levemente cargada hacia mayores de 35 años, especialmente en áreas rurales, y hacia quienes tienen baja escolaridad: el apoyo incondicional al uso de la fuerza alcanza a $23 \%$ entre quienes tienen hasta 8 años de estudio, mientras que entre quienes tienen 13 o más años de estudio o escolaridad superior disminuye a la mitad, solamente $11 \%$, con una diferencia similar en área urbana y rural. Es probable que estos datos no muestren tanto una actitud políticamente relevante hacia el movimiento indígena, sino simplemente una afirmación incondicional del derecho a la tierra que aparece por encima de cualquier consideración.

La identidad étnica mapuche parece estar recluida en la tierra que se constituye en soporte material de la economía mapuche, pero sobre todo en el fundamento simbólico de una comunidad que por otra parte se desvanece totalmente. Como sostén de una identidad étnica, la tierra mapuche no debe ser entendida solamente como propiedad. Es cierto que la tierra no alberga ninguna comunidad, ni existen tierras comunitarias: el 89\% de los mapuches considera que las tierras deben ser de las personas y sus familias como ha sido la costumbre. Pero el valor simbólico y patrimonial de la tierra se manifiesta también en el 65\% de mapuches que aprueba la prohibición ancestral de vender tierras mapuches a no mapuches (hasta $76 \%$ entre mapuches que viven en el campo). El cierre de la comunidad que no se consigue a través de la prohibición del connubio, se logra con la clausura patrimonial de la tierra indígena. Asimismo, mucho de lo que se llama el problema mapuche proviene del desbalance entre la inviabilidad económica de la tierra indígena y la exacerbación de su valor cultural. El minifundio alberga esta contradicción interna: su valor económico no se compadece con su valor simbólico. Las dificultades de afirmar una identidad al margen de la tierra no auguran sino una profundización de este desajuste. 
CUADRO N N $^{\circ}$ 5: AUTOIDENTIFICACIÓN Y SENTIMIENTO DE INTEGRACIÓN NACIONAL ENTRE MAPUCHES SEGÚN CATEGORÍAS DE EDAD

\begin{tabular}{|c|c|c|c|c|c|c|}
\hline & \multicolumn{3}{|c|}{ Jóvenes (18-34) } & \multicolumn{3}{|c|}{ No jóvenes (35 y más) } \\
\hline & $\begin{array}{c}\text { Plenamente } \\
\text { integrado }\end{array}$ & $\begin{array}{l}\text { Parcialmente } \\
\text { integrado }\end{array}$ & $\begin{array}{c}\text { Nada } \\
\text { integrado }\end{array}$ & $\begin{array}{l}\text { Plenamente } \\
\text { integrado }\end{array}$ & $\begin{array}{l}\text { Parcialmente } \\
\text { integrado }\end{array}$ & $\begin{array}{c}\text { Nada } \\
\text { integrado }\end{array}$ \\
\hline \multicolumn{7}{|c|}{ Mapuche urbano } \\
\hline Étnica & 64 & 30 & 6 & 73 & 23 & 4 \\
\hline Mixta & 68 & 27 & 5 & 81 & 17 & 1 \\
\hline Chileno & 83 & 17 & 0 & 84 & 12 & 4 \\
\hline \multicolumn{7}{|c|}{ Mapuche rural } \\
\hline Étnica & 59 & 40 & 1 & 77 & 19 & 4 \\
\hline Mixta & 69 & 31 & 0 & 82 & 14 & 4 \\
\hline Chileno & 40 & 60 & 0 & 75 & 22 & 3 \\
\hline
\end{tabular}

La mirada económica no acierta muchas veces a comprender el significado de la tierra mapuche cuando confunde tierra con propiedad. Tampoco lo hace mejor la mirada política que tiende a confundir tierra con territorio. La encuesta no entrega muchos datos sobre este importante aspecto de la cuestión mapuche. El sentimiento de integración nacional puede ser un buen indicio, sin embargo, del reconocimiento de la nación como unidad territorial: como se ha dicho; el 73\% de los mapuches dice sentirse plenamente integrados al país (apenas 3\% marca nada integrado) y esta proporción es la misma en área rural y urbana. Este sentimiento de integración es algo menor entre quienes se identifican como mapuches a secas (identificación étnica) aunque sólo en la ciudad: en el área rural el sentimiento de integración nacional es tanto o más alto entre quienes se declaran abiertamente mapuches. Los datos indican que identidad étnica no va en desmedro del sentido de pertenencia nacional, lo que puede ser una prueba de que la afirmación étnica de la tierra no tiene un alcance territorial. También los datos que proporciona la encuesta acerca de la exigencia de autonomía van en este mismo sentido: el $80 \%$ de los mapuches considera que las comunidades indígenas “deberían integrarse más al país”, mientras que solamente un $18 \%$ considera que "deberían tener más autonomía del resto de Chile", proporciones que alcanzan a $78 \%$ y $22 \%$ respectivamente entre mapuches de área rural. No parece existir nada parecido a una nación mapuche (y la expresión "pueblo mapuche” es exacta en este sentido, los pueblos tienen tierras, las naciones en cambio tienen dominio jurisdiccional sobre territorios): al menos la encuesta no entrega ningún indicio acerca de 
demandas o aspiraciones propiamente nacionales. La tierra es patrimonio, no territorio: no tiene ningún valor como sede de una organización política, lo que es propio de un pueblo que no ha conocido ninguna forma desarrollada de poder estatal. Por contrapartida, la tierra parece adquirir un valor cultural inconmensurable en el mundo mapuche como soporte de una identidad que ya no encuentra otros espacios ni maneras de afirmarse. 